\title{
Loewy lengths of centers of blocks II
}

\author{
Burkhard Külshammer*, Yoshihiro Otokita ${ }^{\dagger}$ and Benjamin Sambale
}

September 24, 2018

\begin{abstract}
Let $Z B$ be the center of a $p$-block $B$ of a finite group with defect group $D$. We show that the Loewy length $L L(Z B)$ of $Z B$ is bounded by $\frac{|D|}{p}+p-1$ provided $D$ is not cyclic. If $D$ is non-abelian, we prove the stronger bound $L L(Z B)<\min \left\{p^{d-1}, 4 p^{d-2}\right\}$ where $|D|=p^{d}$. Conversely, we classify the blocks $B$ with $L L(Z B) \geq \min \left\{p^{d-1}, 4 p^{d-2}\right\}$. This extends some results previously obtained by the present authors. Moreover, we characterize blocks with uniserial center.
\end{abstract}

Keywords: center of blocks, Loewy length

AMS classification: 20C05, 20C20

\section{Introduction}

The aim of this paper is to extend some results on Loewy lengths of centers of blocks obtained in [8, 11]. In the following we will reuse some of the notation introduced in 8 . In particular, $B$ is a block of a finite group $G$ with respect to an algebraically closed field $F$ of characteristic $p>0$. Moreover, let $D$ be a defect group of $B$. The second author has shown in [11, Corollary 3.3] that the Loewy length of the center of $B$ is bounded by

$$
L L(Z B) \leq|D|-\frac{|D|}{\exp (D)}+1
$$

where $\exp (D)$ is the exponent of $D$. It was already known to Okuyama 9 that this bound is best possible if $D$ is cyclic. The first and the third author have given in [8, Theorem 1] the optimal bound $L L(Z B) \leq L L(F D)$ for blocks with abelian defect groups. Our main result of the present paper establishes the following bound for blocks with non-abelian defect groups:

$$
L L(Z B)<\min \left\{p^{d-1}, 4 p^{d-2}\right\}
$$

where $|D|=p^{d}$. As a consequence we obtain

$$
L L(Z B) \leq p^{d-1}+p-1
$$

for all blocks with non-cyclic defect groups. It can be seen that this bound is optimal whenever $B$ is nilpotent and $D \cong C_{p^{d-1}} \times C_{p}$.

In the second part of the paper we show that $L L(Z B)$ depends more on $\exp (D)$ than on $|D|$. We prove for instance that $L L(Z B) \leq d^{2} \exp (D)$ unless $d=0$. Finally, we use the opportunity to improve a result of Willems [14] about blocks with uniserial center.

In addition to the notation used in the papers cited above, we introduce the following objects. Let $\mathrm{Cl}(G)$ be the set of conjugacy classes of $G$. A $p$-subgroup $P \leq G$ is called a defect group of $K \in \mathrm{Cl}(G)$ if $P$ is a Sylow

\footnotetext{
*Institut für Mathematik, Friedrich-Schiller-Universität, 07743 Jena, Germany, kuelshammer@uni-jena.de

${ }^{\dagger}$ Department of Mathematics and Informatics, Chiba University, Chiba-Shi, 263-8522, Japan, otokita@chiba-u.jp

${ }^{\ddagger}$ Fachbereich Mathematik, TU Kaiserslautern, 67653 Kaiserslautern, Germany, sambale@mathematik.uni-kl.de
} 
$p$-subgroup of $\mathrm{C}_{G}(x)$ for some $x \in K$. Let $\mathrm{Cl}_{P}(G)$ be the set of conjugacy classes with defect group $P$. Let $K^{+}:=\sum_{x \in K} x \in F G$ and

$$
\begin{aligned}
I_{P}(G) & :=\left\langle K^{+}: K \in \mathrm{Cl}_{P}(G)\right\rangle \subseteq Z F G, \\
I_{\leq P}(G) & :=\sum_{Q \leq P} I_{Q}(G) \unlhd Z F G, \\
I_{<P}(G) & :=\sum_{Q<P} I_{Q}(G) \unlhd Z F G .
\end{aligned}
$$

\section{Results}

We begin by restating a lemma of Passman [12, Lemma 2]. For the convenience of the reader we provide a (slightly easier) proof.

Lemma 1 (Passman). Let $P$ be a central p-subgroup of $G$. Then $I_{\leq P}(G) \cdot J Z F G=I_{\leq P}(G) \cdot J F P$.

Proof. Let $K$ be a conjugacy class of $G$ with defect group $P$, and let $x \in K$. Then $P$ is the only Sylow $p$-subgroup of $\mathrm{C}_{G}(x)$, and the $p$-factor $u$ of $x$ centralizes $x$. Thus $u \in P$. Hence $u$ is the $p$-factor of every element in $K$, and $K=u K^{\prime}$ where $K^{\prime}$ is a $p$-regular conjugacy class of $G$ with defect group $P$. This shows that $I:=I_{\leq P}(G)$ is a free $F P$-module with the $p$-regular class sums with defect group $P$ as an $F P$-basis. The canonical epimorphism $\nu: F G \rightarrow F[G / P]$ maps $I$ into $I_{1}(G / P) \subseteq S F[G / P]$. Thus $\nu(I \cdot J Z F G) \subseteq S F[G / P] \cdot J Z F[G / P]=0$. Hence $I \cdot J Z F G \subseteq I \cdot J F P$. The other inclusion is trivial.

Lemma 2. Let $P \leq G$ be a $p$-subgroup of order $p^{n}$. Then

(i) $I_{\leq P}(G) \cdot J Z F G^{L L(F \mathrm{Z}(P))} \subseteq I_{<P}(G)$.

(ii) $I_{\leq P}(G) \cdot J Z F G^{\left(p^{n+1}-1\right) /(p-1)}=0$.

Proof.

(i) Let $\operatorname{Br}_{P}: Z F G \rightarrow Z F \mathrm{C}_{G}(P)$ be the Brauer homomorphism. Since $\operatorname{Ker}\left(\operatorname{Br}_{P}\right) \cap I_{\leq P}(G)=I_{<P}(G)$, we need to show that $\operatorname{Br}_{P}\left(I_{\leq P}(G) \cdot J Z F G^{L L(F \mathrm{Z}(P))}\right)=0$. By Lemma 1] we have

$$
\begin{aligned}
\operatorname{Br}_{P}\left(I_{\leq P}(G) \cdot J Z F G^{L L(F \mathrm{Z}(P))}\right) & \subseteq I_{\leq \mathrm{Z}(P)}\left(\mathrm{C}_{G}(P)\right) \cdot J Z F \mathrm{C}_{G}(P)^{L L(F \mathrm{Z}(P))} \\
& =I_{\leq \mathrm{Z}(P)}\left(\mathrm{C}_{G}(P)\right) \cdot J F \mathrm{Z}(P)^{L L(F \mathrm{Z}(P))}=0 .
\end{aligned}
$$

(ii) We argue by induction on $n$. The case $n=1$ follows from $I_{1}(G) \subseteq S F G$. Now suppose that the claim holds for $n-1$. Since $L L(F \mathrm{Z}(P)) \leq|P|=p^{n}$, (i) implies

$$
\begin{aligned}
I_{\leq P}(G) \cdot J Z F G^{\left(p^{n+1}-1\right) /(p-1)} & =I_{\leq P}(G) \cdot J Z F G^{p^{n}} J Z F G^{\left(p^{n}-1\right) /(p-1)} \\
& \subseteq I_{<P}(G) \cdot J Z F G^{\left(p^{n}-1\right) /(p-1)} \\
& =\sum_{Q<P} I_{\leq Q}(G) \cdot J Z F G^{\left(p^{n}-1\right) /(p-1)}=0 .
\end{aligned}
$$

Recall from [8, Lemma 9] the following group

$$
W_{p^{d}}:=\left\langle x, y, z \mid x^{p^{d-2}}=y^{p}=z^{p}=[x, y]=[x, z]=1,[y, z]=x^{p^{d-3}}\right\rangle .
$$

Note that $W_{p^{d}}$ is a central product of $C_{p^{d-2}}$ and an extraspecial group of order $p^{3}$. Now we prove our main theorem which improves [8, Theorem 12].

Theorem 3. Let $B$ be a block of FG with non-abelian defect group $D$ of order $p^{d}$. Then one of the following holds 
(i) $L L(Z B)<3 p^{d-2}$.

(ii) $p \geq 5, D \cong W_{p^{d}}$ and $L L(Z B)<4 p^{d-2}$.

In any case we have

$$
L L(Z B)<\min \left\{p^{d-1}, 4 p^{d-2}\right\}
$$

Proof. By [8, Proposition 15], we may assume that $p>2$. Since $D$ is non-abelian, $|D: \mathrm{Z}(D)| \geq p^{2}$ and $L L(F \mathrm{Z}(D)) \leq p^{d-2}$. Let $Q$ be a maximal subgroup of $D$. If $Q$ is cyclic, then $D \cong M_{p^{n}}$ and the claim follows from [8, Proposition 10]. Hence, we may assume that $Q$ is not cyclic. Then $L L(F \mathrm{Z}(Q)) \leq p^{d-2}+p-1$. Now setting $\lambda:=\frac{p^{d-1}-1}{p-1}$ it follows from Lemma 2 that

$$
\begin{aligned}
J Z B^{2 p^{d-2}+p-1+\lambda} & \subseteq 1_{B} J Z F G^{2 p^{d-2}+p-1+\lambda} \subseteq I_{\leq D}(G) \cdot J Z F G^{2 p^{d-2}+p-1+\lambda} \\
& \subseteq I_{<D}(G) \cdot J Z F G^{p^{d-2}+p-1+\lambda}=\sum_{Q<D} I_{\leq Q}(G) \cdot J Z F G^{p^{d-2}+p-1+\lambda} \\
& \subseteq \sum_{Q<D} I_{<Q}(G) \cdot J Z F G^{\lambda}=0 .
\end{aligned}
$$

Since $2 p^{d-2}+p-1+\lambda \leq 4 p^{d-2}$, we are done in case $p \geq 5$ and $D \cong W_{p^{d}}$. If $p=3$ and $D \cong W_{p^{d}}$, then the claim follows from [8, Lemma 11]. Now suppose that $D \neq W_{p^{d}}$. If $\mathrm{Z}(D)$ is cyclic of order $p^{d-2}$, then the claim follows from [8, Lemma 9 and Proposition 10]. Hence, suppose that $\mathrm{Z}(D)$ is non-cyclic or $|\mathrm{Z}(D)|<p^{d-2}$. Then $d \geq 4$ and $L L(F \mathrm{Z}(D)) \leq p^{d-3}+p-1$. The arguments above give $L L(Z B) \leq p^{d-2}+p^{d-3}+2 p-2+\lambda$, hence we are done whenever $p>3$.

In the following we assume that $p=3$. Here we have $L L(Z B) \leq 3^{d-2}+3^{d-3}+4+\frac{1}{2}\left(3^{d-1}-1\right)$ and it suffices to handle the case $d=4$. By [11, Theorem 3.2], there exists a non-trivial $B$-subsection $(u, b)$ such that

$$
L L(Z B) \leq(|\langle u\rangle|-1) L L(Z \bar{b})+1
$$

where $\bar{b}$ is the unique block of $F \mathrm{C}_{G}(u) /\langle u\rangle$ dominated by $b$. We may assume that $\bar{b}$ has defect group $\mathrm{C}_{D}(u) /\langle u\rangle$ (see [13, Lemma 1.34]). If $u \notin \mathrm{Z}(D)$, we obtain $L L(Z B)<\left|\mathrm{C}_{D}(u)\right| \leq 27$ as desired. Hence, let $u \in \mathrm{Z}(D)$. Then $D /\langle u\rangle$ is not cyclic. Moreover, by our assumption on $\mathrm{Z}(D)$, we have $|\langle u\rangle|=3$. Now it follows from [8, Theorem 1 , Proposition 10 and Lemma 11] applied to $\bar{b}$ that

$$
L L(Z B) \leq 2 L L(Z \bar{b})+1 \leq 23<27 .
$$

We do not expect that the bounds in Theorem 3 are sharp. In fact, we do not know if there are $p$-blocks $B$ with non-abelian defect groups of order $p^{d}$ such that $p>2$ and $L L(Z B)>p^{d-2}$. See also Proposition 7 below.

Corollary 4. Let $B$ be a block of $F G$ with non-cyclic defect group of order $p^{d}$. Then

$$
L L(Z B) \leq p^{d-1}+p-1 .
$$

Proof. By Theorem 3, we may assume that $B$ has abelian defect group $D$. Then [8, Theorem 1] implies $L L(Z B) \leq L L(F D) \leq p^{d-1}+p-1$.

We are now in a position to generalize [8, Corollary 16].

Corollary 5. Let $B$ be a block of $F G$ with defect group $D$ of order $p^{d}$ such that $L L(Z B) \geq \min \left\{p^{d-1}, 4 p^{d-2}\right\}$. Then one of the following holds

(i) $D$ is cyclic.

(ii) $D \cong C_{p^{d-1}} \times C_{p}$.

(iii) $D \cong C_{2} \times C_{2} \times C_{2}$ and $B$ is nilpotent. 
Proof. Again by Theorem 3 we may assume that $D$ is abelian. By [8, Corollary 16], we may assume that $p>2$. Suppose that $D$ is of type $\left(p^{a_{1}}, \ldots, p^{a_{s}}\right)$ such that $s \geq 3$. Then

$$
\begin{aligned}
\min \left\{p^{d-1}, 4 p^{d-2}\right\} & \leq L L(Z B)=p^{a_{1}}+\ldots+p^{a_{s}}-s+1 \\
& \leq p^{a_{1}}+p^{a_{2}}+p^{a_{3}+\ldots+a_{s}}-2 \leq p^{d-2}+2(p-1) .
\end{aligned}
$$

This clearly leads to a contradiction. Therefore, $s \leq 2$ and the claim follows.

In case (i) of Corollary 5 it is known conversely that $L L(Z B)=\frac{p^{d}-1}{l(B)}+1>p^{d-1}$ (see [6, Corollary 2.8]).

Our next result gives a more precise bound by invoking the exponent of a defect group.

Theorem 6. Let $B$ be a block of FG with defect group $D$ of order $p^{d}>1$ and exponent $p^{e}$. Then

$$
L L(Z B) \leq\left(\frac{d}{e}+1\right)\left(\frac{d}{2}+\frac{1}{p-1}\right)\left(p^{e}-1\right) .
$$

In particular, $L L(Z B) \leq d^{2} p^{e}$.

Proof. Let $\alpha:=\lfloor d / e\rfloor$. Let $P \leq D$ be abelian of order $p^{i e+j}$ with $0 \leq i \leq \alpha$ and $0 \leq j<e$. If $P$ has type $\left(p^{a_{1}}, \ldots, p^{a_{r}}\right)$, then $a_{i} \leq e$ for $i=1, \ldots, r$ and

$$
L L(F P)=\left(p^{a_{1}}-1\right)+\ldots+\left(p^{a_{r}}-1\right)+1 \leq i\left(p^{e}-1\right)+p^{j} .
$$

Arguing as in Theorem 3, we obtain

$$
\begin{aligned}
L L(Z B) & \leq \sum_{i=0}^{\alpha} \sum_{j=0}^{e-1} i\left(p^{e}-1\right)+p^{j}=e\left(p^{e}-1\right)\left(\sum_{i=0}^{\alpha} i\right)+(\alpha+1) \frac{p^{e}-1}{p-1} \\
& =e\left(p^{e}-1\right) \frac{\alpha(\alpha+1)}{2}+(\alpha+1) \frac{p^{e}-1}{p-1} \\
& \leq\left(\frac{d}{e}+1\right)\left(\frac{d}{2}+\frac{1}{p-1}\right)\left(p^{e}-1\right) .
\end{aligned}
$$

This proves the first claim. For the second claim we note that

$$
\left(\frac{d}{e}+1\right)\left(\frac{d}{2}+\frac{1}{p-1}\right) \leq(d+1)\left(\frac{d}{2}+1\right) \leq d^{2}
$$

unless $d \leq 3$. In these small cases the claim follows from Theorem 3 and Corollary 4

If $2 e>d$ and $p$ is large, then the bound in Theorem 6 is approximately $d p^{e}$. The groups of the form $G=D=$ $C_{p^{e}} \times \ldots \times C_{p^{e}}$ show that there is no bound of the form $L L(Z B) \leq C p^{e}$ where $C$ is an absolute constant. A more careful argumentation in the proof above gives the stronger (but opaque) bound

$$
L L(Z B) \leq \alpha\left(p^{e}-1\right)\left(\frac{e(\alpha-1)}{2}+\frac{1}{p-1}+d-\alpha e\right)+\beta\left(p^{e}-1\right)+\frac{p^{d-\alpha e}-1}{p-1}+p^{d-2-\beta e}
$$

for non-abelian defect groups where $\alpha:=\left\lfloor\frac{d-1}{e}\right\rfloor$ and $\beta:=\left\lfloor\frac{d-2}{e}\right\rfloor$. We omit the details.

In the next result we compute the Loewy length for $d=e+1$.

Proposition 7. Let $B$ be a block of FG with non-abelian defect group of order $p^{d}$ and exponent $p^{d-1}$. Then

$$
L L(Z B) \leq \begin{cases}2^{d-2}+1 & \text { if } p=2 \\ p^{d-2} & \text { if } p>2\end{cases}
$$

and both bounds are optimal for every $d \geq 3$. 
Proof. Let $D$ be a defect group of $B$. If $p>2$, then $D \cong M_{p^{d}}$ and we have shown $L L(Z B) \leq p^{d-2}$ in $[8$, Proposition 10]. Equality holds if and only if $B$ is nilpotent.

Therefore, we may assume $p=2$ in the following. The modular groups $M_{2^{d}}$ are still handled by [8, Proposition 10]. Hence, it remains to consider the defect groups of maximal nilpotency class, i. e. $D \in\left\{D_{2^{d}}, Q_{2^{d}}, S D_{2^{d}}\right\}$. By [8, Proposition 10], we may assume that $d \geq 4$. The isomorphism type of $Z B$ is uniquely determined by $D$ and the fusion system of $B$ (see [2]). The possible cases are listed in [13, Theorem 8.1]. If $B$ is nilpotent, [8, Proposition 8] gives $L L(Z B)=L L(Z F D) \leq L L\left(F D^{\prime}\right)=2^{d-2}$. Moreover, in the case $D \cong D_{2^{d}}$ and $l(B)=3$ we have $L L(Z B) \leq k(B)-l(B)+1=2^{d-2}+1$ by [11, Proposition 2.2]. In the remaining cases we present $B$ by quivers with relations which were constructed originally by Erdmann [3]. We refer to [4, Appendix B].

(i) $D \cong D_{2^{d}}, l(B)=2$ :

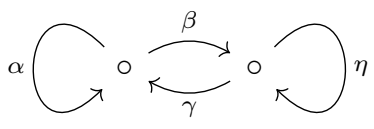

$$
\begin{gathered}
\beta \eta=\eta \gamma=\gamma \beta=\alpha^{2}=0, \\
\alpha \beta \gamma=\beta \gamma \alpha, \\
\eta^{2^{d-2}}=\gamma \alpha \beta .
\end{gathered}
$$

By [4, Lemma 2.3.3], we have

$$
Z B=\operatorname{span}\left\{1, \beta \gamma, \alpha \beta \gamma, \eta^{i}: i=1, \ldots, 2^{d-2}\right\}
$$

It follows that $J Z B^{2}=\left\langle\eta^{2}\right\rangle$ and $L L(Z B)=2^{d-2}+1$.

(ii) $D \cong Q_{2^{d}}, l(B)=2$ : Here [15, Lemma 6] gives the isomorphism type of $Z B$ directly as a quotient of a polynomial ring

$$
Z B \cong F[U, Y, S, T] /\left(Y^{2^{d-2}+1}, U^{2}-Y^{2^{d-2}}, S^{2}, T^{2}, S Y, S U, S T, U Y, U T, Y T\right) .
$$

It follows that $J Z B^{2}=\left(Y^{2}\right)$ and again $L L(Z B)=2^{d-2}+1$.

(iii) $D \cong Q_{2^{d}}, l(B)=3$ :

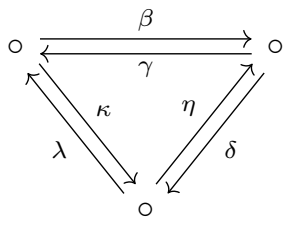

$$
\begin{gathered}
\beta \delta=(\kappa \lambda)^{2^{d-2}-1} \kappa, \eta \gamma=(\lambda \kappa)^{2^{d-2}-1} \lambda, \\
\delta \lambda=\gamma \beta \gamma, \kappa \eta=\beta \gamma \beta, \lambda \beta=\eta \delta \eta, \\
\gamma \kappa=\delta \eta \delta, \gamma \beta \delta=\delta \eta \gamma=\lambda \kappa \eta=0 .
\end{gathered}
$$

By [4, Lemma 2.5.15],

$$
Z B=\operatorname{span}\left\{1, \beta \gamma+\gamma \beta,(\kappa \lambda)^{i}+(\lambda \kappa)^{i}, \delta \eta+\eta \delta,(\beta \gamma)^{2},(\lambda \kappa)^{2^{d-2}},(\delta \eta)^{2}: i=1, \ldots, 2^{d-2}-1\right\}
$$

We compute

$$
\begin{aligned}
(\beta \gamma+\gamma \beta)^{2}=(\beta \gamma)^{2}+(\gamma \beta)^{2} & =(\beta \gamma)^{2}+\delta \lambda \beta=(\beta \gamma)^{2}+(\delta \eta)^{2} \\
(\beta \gamma+\gamma \beta)(\kappa \lambda+\lambda \kappa) & =\beta \gamma \kappa \lambda=\beta \delta \eta \delta \lambda=\beta \delta \eta \gamma \beta \gamma=0 \\
(\beta \gamma+\gamma \beta)(\delta \eta+\eta \delta) & =\gamma \beta \delta \eta=0 \\
(\beta \gamma+\gamma \beta)(\beta \gamma)^{2} & =(\beta \gamma)^{3}=\beta \gamma \beta \delta \lambda=0 \\
(\beta \gamma+\gamma \beta)(\lambda \kappa)^{2^{d-2}} & =0 \\
(\beta \gamma+\gamma \beta)(\delta \eta)^{2} & =\gamma \beta \delta \eta \delta \eta=0 \\
\left((\kappa \lambda)^{2^{d-2}-1}+(\lambda \kappa)^{2^{d-2}-1}\right)(\kappa \lambda+\lambda \kappa) & =\kappa \eta \gamma+(\lambda \kappa)^{2^{d-2}}=(\beta \gamma)^{2}+(\lambda \kappa)^{2^{d-2}} \\
(\kappa \lambda+\lambda \kappa)(\delta \eta+\eta \delta) & =\lambda \kappa \eta \delta=0 \\
(\kappa \lambda+\lambda \kappa)(\beta \gamma)^{2} & =\kappa \lambda \beta \gamma \beta \gamma=\kappa \eta \delta \eta \gamma \beta \gamma=0 \\
(\kappa \lambda+\lambda \kappa)(\lambda \kappa)^{2^{d-2}} & =\lambda \kappa \eta \gamma \kappa=0
\end{aligned}
$$




$$
\begin{aligned}
(\kappa \lambda+\lambda \kappa)(\delta \eta)^{2} & =0, \\
(\delta \eta+\eta \delta)^{2}=(\delta \eta)^{2}+(\eta \delta)^{2} & =(\delta \eta)^{2}+\lambda \beta \delta=(\delta \eta)^{2}+(\lambda \kappa)^{2^{d-2}}, \\
(\delta \eta+\eta \delta)(\beta \gamma)^{2} & =0, \\
(\delta \eta+\eta \delta)(\lambda \kappa)^{2^{d-2}} & =\eta \delta(\lambda \kappa)^{2^{d-2}}=\eta \delta \eta \gamma \kappa=0, \\
(\delta \eta+\eta \delta)(\delta \eta)^{2} & =\delta \lambda \beta \delta \eta=\gamma \beta \gamma \beta \delta \eta=0, \\
(\beta \gamma)^{2}(\beta \gamma)^{2} & =(\beta \gamma)^{2}(\lambda \kappa)^{2^{d-2}}=(\beta \gamma)^{2}(\delta \eta)^{2}=0, \\
(\lambda \kappa)^{2^{d-2}}(\lambda \kappa)^{2^{d-2}} & =(\lambda \kappa)^{2^{d-2}}(\delta \eta)^{2}=0, \\
(\delta \eta)^{2}(\delta \eta)^{2} & =\gamma \kappa \eta(\delta \eta)^{2}=\gamma \beta \gamma \beta(\delta \eta)^{2}=0 .
\end{aligned}
$$

Hence, $J Z B^{2}=\left\langle(\lambda \kappa)^{2}+(\kappa \lambda)^{2},(\beta \gamma)^{2}+(\delta \eta)^{2}\right\rangle$ and $J Z B^{3}=\left\langle(\lambda \kappa)^{3}+(\kappa \lambda)^{3}\right\rangle$. This implies $L L(Z B)=$ $2^{d-2}+1$.

(iv) $D \cong S D_{2^{d}}, k(B)=2^{d-2}+3$ and $l(B)=2$ :

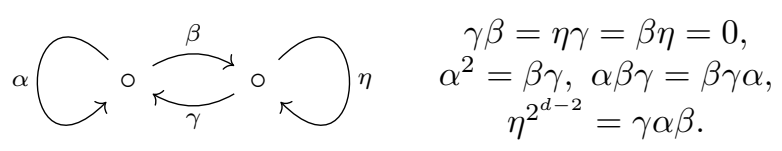

By [5, Section 5.1], we have

$$
Z B=\operatorname{span}\left\{1, \beta \gamma, \alpha \beta \gamma, \eta^{i}: i=1, \ldots, 2^{d-2}\right\} .
$$

As in (i) we obtain $J Z B^{2}=\left\langle\eta^{2}\right\rangle$ and $L L(Z B)=2^{d-2}+1$.

(v) $D \cong S D_{2^{d}}, k(B)=2^{d-2}+4$ and $l(B)=2$ :

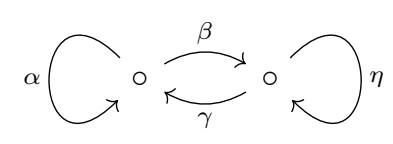

$$
\begin{gathered}
\beta \eta=\alpha \beta \gamma \alpha \beta, \gamma \beta=\eta^{2^{d-2}-1} \\
\eta \gamma=\gamma \alpha \beta \gamma \alpha \\
\beta \eta^{2}=\eta^{2} \gamma=\alpha^{2}=0
\end{gathered}
$$

By [5, Section 5.2.2], we have

$$
Z B=\operatorname{span}\left\{1, \alpha \beta \gamma+\beta \gamma \alpha+\gamma \alpha \beta, \beta \gamma \alpha \beta \gamma,(\alpha \beta \gamma)^{2}, \eta^{i}, \eta+\alpha \beta \gamma \alpha: i=2, \ldots, 2^{d-2}\right\} .
$$

Since $(\alpha \beta \gamma)^{2}=\beta \eta \gamma=(\beta \gamma \alpha)^{2}$ and $(\gamma \alpha \beta)^{2}=\eta \gamma \beta=\eta^{2^{d-2}}$, it follows that

$$
(\alpha \beta \gamma+\beta \gamma \alpha+\gamma \alpha \beta)^{2}=(\alpha \beta \gamma)^{2}+(\beta \gamma \alpha)^{2}+(\gamma \alpha \beta)^{2}=\eta^{2^{d-2}} .
$$

Similarly,

$$
\begin{aligned}
(\alpha \beta \gamma+\beta \gamma \alpha+\gamma \alpha \beta) \beta \gamma \alpha \beta \gamma & =0, \\
(\alpha \beta \gamma+\beta \gamma \alpha+\gamma \alpha \beta)(\alpha \beta \gamma)^{2} & =0, \\
(\alpha \beta \gamma+\beta \gamma \alpha+\gamma \alpha \beta) \eta^{2} & =0, \\
(\alpha \beta \gamma+\beta \gamma \alpha+\gamma \alpha \beta)(\eta+\alpha \beta \gamma \alpha) & =0, \\
(\beta \gamma \alpha \beta \gamma)^{2} & =0, \\
\beta \gamma \alpha \beta \gamma(\alpha \beta \gamma)^{2} & =0, \\
\beta \gamma \alpha \beta \gamma \eta^{2}=\beta \gamma \alpha \beta \eta^{2} \gamma & =0, \\
\beta \gamma \alpha \beta \gamma(\eta+\alpha \beta \gamma \alpha)=\beta \gamma(\alpha \beta \gamma)^{2} \alpha & =0, \\
(\alpha \beta \gamma)^{2}(\alpha \beta \gamma)^{2} & =0,
\end{aligned}
$$




$$
\begin{aligned}
(\alpha \beta \gamma)^{2} \eta^{2} & =0, \\
(\alpha \beta \gamma)^{2}(\eta+\alpha \beta \gamma \alpha) & =0, \\
\eta^{2}(\eta+\alpha \beta \gamma \alpha) & =\eta^{3}, \\
(\eta+\alpha \beta \gamma \alpha)^{2} & =\eta^{2} .
\end{aligned}
$$

Consequently, $J Z B^{2}=\left\langle\eta^{2}\right\rangle$ and $L L(Z B)=2^{d-2}+1$.

(vi) $D \cong S D_{2^{d}}, l(B)=3$ :

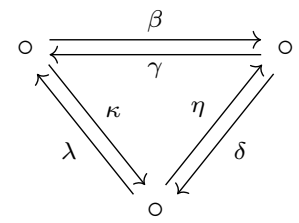

$$
\begin{gathered}
\kappa \eta=\eta \gamma=\gamma \kappa=0, \delta \lambda=(\gamma \beta)^{2^{d-2}-1} \gamma, \\
\beta \delta=\kappa \lambda \kappa, \lambda \beta=\eta .
\end{gathered}
$$

From [4, Lemma 2.4.16] we get

$$
Z B=\operatorname{span}\left\{1,(\beta \gamma)^{i}+(\gamma \beta)^{i}, \kappa \lambda+\lambda \kappa,(\beta \gamma)^{2^{d-2}},(\lambda \kappa)^{2}, \delta \eta: i=1, \ldots, 2^{d-2}-1\right\} .
$$

We compute

$$
\begin{aligned}
(\beta \gamma+\gamma \beta)\left((\beta \gamma)^{2^{d-2}-1}+(\gamma \beta)^{2^{d-2}-1}\right) & =(\beta \gamma)^{2^{d-2}}+\delta \lambda \beta=(\beta \gamma)^{2^{d-2}}+\delta \eta, \\
(\beta \gamma+\gamma \beta)(\kappa \lambda+\lambda \kappa) & =\beta \gamma \kappa \lambda=0, \\
(\beta \gamma+\gamma \beta)(\beta \gamma)^{2^{d-2}} & =\beta \delta \lambda \beta \gamma=\kappa \lambda \kappa \eta \gamma=0, \\
(\beta \gamma+\gamma \beta)(\lambda \kappa)^{2} & =0, \\
(\beta \gamma+\gamma \beta) \delta \eta & =\gamma \beta \delta \eta=\gamma \kappa \lambda \kappa \eta=0, \\
(\kappa \lambda+\lambda \kappa)^{2} & =\beta \delta \lambda+(\lambda \kappa)^{2}=(\beta \gamma)^{2^{d-2}}+(\lambda \kappa)^{2}, \\
(\kappa \lambda+\lambda \kappa)(\beta \gamma)^{2^{d-2}} & =\kappa \lambda \beta \gamma(\beta \gamma)^{2^{d-2}-1}=\kappa \eta \gamma(\beta \gamma)^{2^{d-2}-1}=0, \\
(\kappa \lambda+\lambda \kappa)(\lambda \kappa)^{2} & =\lambda(\beta \gamma)^{2^{d-2}} \kappa=\eta \gamma(\beta \gamma)^{2^{d-2}-1} \kappa=0, \\
(\kappa \lambda+\lambda \kappa) \delta \eta & =0, \\
(\beta \gamma)^{2^{d-2}}(\beta \gamma)^{2^{d-2}} & =(\beta \gamma)^{2^{d-2}}(\lambda \kappa)^{2}=(\beta \gamma)^{2^{d-2}} \delta \eta=0, \\
(\lambda \kappa)^{2}(\lambda \kappa)^{2} & =(\lambda \kappa)^{2} \delta \eta=0, \\
(\delta \eta)^{2} & =\delta \lambda \beta \delta \eta=\delta \lambda \kappa \lambda \kappa \eta=0 .
\end{aligned}
$$

Hence, $J Z B^{2}=\left\langle(\beta \gamma)^{2}+(\gamma \beta)^{2},(\kappa \lambda)^{2}+\delta \eta\right\rangle$ and $J Z B^{3}=\left\langle(\beta \gamma)^{3}+(\gamma \beta)^{3}\right\rangle$. This implies $L L(Z B)=$ $2^{d-2}+1$.

It is interesting to note the difference between even and odd primes in Proposition 7, For $p=2$, non-nilpotent blocks gives larger Loewy lengths while for $p>2$ the maximal Loewy length is only assumed for nilpotent blocks.

Recall that a lower defect group of a block $B$ of $F G$ is a $p$-subgroup $Q \leq G$ such that

$$
I_{<Q}(G) 1_{B} \neq I_{\leq Q}(G) 1_{B} .
$$

In this case $Q$ is conjugate to a subgroup of a defect group $D$ of $B$ and conversely $D$ is also a lower defect group since $1_{B} \in I_{\leq D}(G) \backslash I_{<D}(G)$. It is clear that in the proofs of Theorem 3 and Theorem 6 it suffices to sum over the lower defect groups of $B$. In particular there exists a chain of lower defect groups $Q_{1}<\ldots<Q_{n}=D$ such that $L L(Z B) \leq \sum_{i=1}^{n} L L\left(F \mathrm{Z}\left(Q_{i}\right)\right)$. Unfortunately, it is hard to compute the lower defect groups of a given block.

The following proposition generalizes [14, Theorem 1.5]. 
Proposition 8. Let $B$ be a block of $F G$. Then $Z B$ is uniserial if and only if $B$ is nilpotent with cyclic defect groups.

Proof. Suppose first that $Z B$ is uniserial. Then $Z B \cong F[X] /\left(X^{n}\right)$ for some $n \in \mathbb{N}$; in particular, $Z B$ is a symmetric $F$-algebra. Then [10, Theorems 3 and 5] implies that $B$ is nilpotent with abelian defect group $D$. Thus, by a result of Broué and Puig [1] (see also [7]), $B$ is Morita equivalent to $F D$; in particular, $F D$ is also uniserial. Thus $D$ is cyclic.

Conversely, suppose that $B$ is nilpotent with cyclic defect group $D$. Then the Broué-Puig result mentioned above implies that $B$ is Morita equivalent of $F D$. Thus $Z B \cong Z F D=F D$. Since $F D$ is uniserial, the result follows.

A similar proof shows that $Z B$ is isomorphic to the group algebra of the Klein four group over an algebraically closed field of characteristic 2 if and only if $B$ is nilpotent with Klein four defect groups.

\section{Acknowledgment}

The third author is supported by the German Research Foundation (project SA 2864/1-1) and the Daimler and Benz Foundation (project 32-08/13).

\section{References}

[1] M. Broué and L. Puig, A Frobenius theorem for blocks, Invent. Math. 56 (1980), 117-128.

[2] M. Cabanes and C. Picaronny, Types of blocks with dihedral or quaternion defect groups, J. Fac. Sci. Univ. Tokyo Sect. IA Math. 39 (1992), 141-161. Revised version: http://www.math.jussieu.fr/ ${ }^{\sim}$ cabanes/type99.pdf.

[3] K. Erdmann, Blocks of tame representation type and related algebras, Lecture Notes in Math., Vol. 1428, Springer-Verlag, Berlin, 1990.

[4] T. Holm, Blocks of Tame Representation Type and Related Algebras: Derived Equivalences and Hochschild Cohomology, Habilitationsschrift, Magdeburg, 2001.

[5] T. Holm and A. Zimmermann, Generalized Reynolds ideals and derived equivalences for algebras of dihedral and semidihedral type, J. Algebra 320 (2008), 3425-3437.

[6] S. Koshitani, B. Külshammer and B. Sambale, On Loewy lengths of blocks, Math. Proc. Cambridge Philos. Soc. 156 (2014), 555-570.

[7] B. Külshammer, On the structure of block ideals in group algebras of finite groups, Comm. Algebra 8 (1980), $1867-1872$.

[8] B. Külshammer and B. Sambale, Loewy lengths of centers of blocks, submitted.

[9] T. Okuyama, On the radical of the center of a group algebra, Hokkaido Math. J. 10 (1981), 406-408.

[10] T. Okuyama and Y. Tsushima, Local properties of p-block algebras of finite groups, Osaka J. Math. 20 (1983), 33-41.

[11] Y. Otokita, Characterizations of blocks by Loewy lengths of their centers, to appear in Proc. Amer. Math. Soc., DOI: $10.1090 /$ proc/13529.

[12] D. S. Passman, The radical of the center of a group algebra, Proc. Amer. Math. Soc. 78 (1980), 323-326.

[13] B. Sambale, Blocks of finite groups and their invariants, Springer Lecture Notes in Math., Vol. 2127, Springer-Verlag, Cham, 2014. 
[14] W. Willems, The representation type of the centre of a group algebra, J. London Math. Soc. (2) 33 (1986), 253-259.

[15] A. Zimmermann, Külshammer ideals of algebras of quaternion type, arXiv:1605.07757v2, 